\title{
AVALIAÇÃO DOS EFEITOS DA RADIAÇÃO GAMA NA CONSERVAÇÃO DA QUALIDADE DA POLPA DE AMORA-PRETA (Rubus spp. L.) ${ }^{1}$
}

\author{
MICHELE PAULA DA SILVA ${ }^{2}$, FLAVIA DOS SANTOS GOMES ${ }^{3}$, \\ MURILLO FREIRE JUNIOR ${ }^{4}$, LOURDES MARIA CORREA CABRAL ${ }^{5}$
}

RESUMO - A amora-preta é uma fruta que vem despertando a atenção de produtores e consumidores devido ao seu sabor agradável, cor atrativa e por apresentar em sua composição elevado teor de compostos bioativos. O grande entrave para consumo e a comercialização dos frutos da amoreira é sua elevada taxa respiratória, o que reduz sua vida útil. Uma alternativa viável para o aproveitamento econômico dessas frutas consiste em sua industrialização, podendo ser congeladas, enlatadas, processadas na forma de polpa, ou na forma de sucos e geleias. O objetivo deste trabalho foi avaliar os efeitos da radiação gama na conservação da polpa de amora. A irradiação foi realizada no laboratório de irradiação gama do Centro de Desenvolvimento da Tecnologia Nuclear (CDTN), em uma fonte de cobalto 60, com as seguintes doses de radiação: 0,75 kGy; 1,5 kGy e $3 \mathrm{kGy}$, sob uma taxa de dose de 3,24 kGy.h-1. A polpa não irradiada foi utilizada como controle. As polpas de amora irradiadas foram armazenadas à temperatura de $4^{\circ} \mathrm{C}$, sendo avaliadas nos tempos de 0 ; 7; 15; 30 e 60 dias. Para verificar os efeitos da radiação gama no processamento na polpa, foi realizada a caracterização físico-química e química através das análises de acidez, pH, sólidos solúveis, sólidos totais, teor de antocianinas, atividade antioxidante e cor. Também foi feita análise microbiológica de acordo com a legislação brasileira vigente. O processo de irradiação aumentou a vida útil de prateleira da polpa em até 60 dias, sendo que o tratamento com uma dose de 1,5 kGy foi a que proporcionou a melhor qualidade microbiológica.

Termos para indexação: Antocianinas, atividade antioxidante, Rubus spp., vida útil de prateleira, radiação gama.

\section{EVALUATION OF GAMMA RADIATION PROCESS ON THE QUALITY OF BLACKBERRY (RUBUS SPP. L.) PULP}

\begin{abstract}
The blackberry is a fruit that has attracted the attention of producers and consumers due to its pleasant flavor, attractive color and composition present in high content of bioactive compounds. The major deterrent to consumption and marketing of the blackberry fruits is its high respiration rate, which reduces its lifetime. A viable alternative to the economic utilization of these fruits is its industrialization, can be frozen, canned, processed in pulp form, or in the form of juices and jellies. The objective of this study was to evaluate the effects of gamma radiation on the conservation of blackberry pulp. The irradiation was performed in the laboratory of gamma irradiation in the Development Center of Nuclear Technology (CDTN) in a cobalt 60 source, with the following radiation doses : $0.75 \mathrm{kGy} ; 1.5 \mathrm{kGy}$ and $3 \mathrm{kGy}$, under a dose rate of $3.24 \mathrm{kGy} \cdot \mathrm{h}^{-1}$. The non-irradiated pulp was used as control. The irradiated blackberry pulps were stored at temperature of $4{ }^{\circ} \mathrm{C}$ and evaluated at $0,7,15,30$ and 60 days. To check the effects of gamma radiation on the pulp processing, it was realized physicochemical and chemical characterization through the analysis of acidity, $\mathrm{pH}$, soluble solids, total solids, anthocyanins, antioxidant activity and color. Microbiological analysis according to Brazilian legislation was also done. The irradiation process increase the shelf life of the pulp within 60 days, and the treatment with a dose of $1.5 \mathrm{kGy}$ was the one that provided better microbiological quality.
\end{abstract}

Index terms: Anthocyanins, antioxidant activity, shelf life, gamma irradiation and Rubus spp.

\footnotetext{
1(Trabalho 218-13). Recebido em: 20-05-2013. Aceito para publicação em: 09-05-2014.

${ }^{2}$ Departamento de Tecnologia de Alimentos, Universidade Federal Rural do Rio de Janeiro. E-mail: michelepauladasilva@yahoo.com.br ${ }^{3}$ Embrapa Agroindústria de Alimentos. E-mail: flavia.gomes@embrapa.br

${ }^{4}$ Embrapa Agroindústria de Alimentos. E-mail: murillo.freire@embrapa.br

${ }^{5}$ Embrapa Agroindústria de Alimentos. E-mail: lourdes.cabral@embrapa.br
}

Rev. Bras. Frutic., Jaboticabal - SP, v. 36, n. 3, p. 620- 627, Setembro 2014 


\section{INTRODUÇÃO}

Dentre as várias frutas de clima temperado está a amora (Rubus spp.),com boas perspectivas de cultivo e comercialização. A introdução dessa cultura no Brasil ocorreu no Rio Grande do Sul, pela Embrapa, em 1972. Logo em seguida, a cultura foi sendo difundida para o sul de Minas Gerais, São Paulo, Paraná e Santa Catarina (SCHAKER;ANTONIOLLI, 2009).

A amora-preta é uma fruta que pode ser destinada tanto para o consumo in natura quanto para elaboração de produtos industrializados (BOWENFORBES et al., 2010). Tais características têm estimulado tanto o consumo do suco integral, como a produção de suco concentrado como ingrediente para iogurte, sorvete e produtos geleificados, como geleias e doces cremosos (WU et al., 2010).

Essa fruta vem despertando a atenção dos consumidores e produtores por apresentar em sua composição elevado teor de compostos bioativos. Há na literatura relatos que esses compostos apresentam propriedades benéficas, tais como: a redução do risco de câncer e de doenças cardiovasculares (BOWENFORBES et al., 2010).

A produção de amora apresenta uma grande limitação quanto ao atendimento ao mercado de fruta fresca. Por ser uma fruta que apresenta grande fragilidade e elevada perecibilidade, sua vida útil é reduzida, restringindo seu consumo (ANTUNES et al., 2006). O processamento da amora na forma de polpa apresenta-se com uma alternativa para esta cultura, disponibilizando para o consumidor um produto pronto para ser utilizado sob diferentes formas (JACQUES et al., 2010).

As frutas in natura apresentam elevada população de microrganismos, por isso a necessidade de a mesma passar por algum processamento, pois dessa forma irá inativar esse microrganismo, eliminando a chance de ocasionar surtos nos consumidores. A principal exigência é que um processo deve assegurar a segurança microbiana do produto, preservando as características sensoriais e nutricionais para a obtenção de produtos semelhante à matéria-prima (SONG et al., 2007).

A irradiação gama é método de conservação a frio, que proporciona durabilidade muitas vezes maior do que a pasteurização, pois não influencia na aparência e na composição do alimento (FARKAS, 2006). A Food Agriculture Organization (FAO), Organização Mundial de Saúde (OMS) e a Agência Internacional de Energia Atômica (IAEA) concluíram em um relatório elaborado em 1981 que a irradiação de alimentos até a dosagem de $10 \mathrm{kGy}$ não resulta em danos toxicológicos e não oferece riscos (FARKAS;MOHÁCSI-FARKAS, 2011).

A radiação ionizante é um processo comprovadamente seguro e tem sido avaliado em muitas aplicações. Em função da dose aplicada no alimento, observa-se melhoria na qualidade microbiológica do produto, resultando na redução de perdas no armazenamento e prolongamento da vida útil (ALAM KHAN;ABRAHEM, 2010).

Este trabalho teve por objetivo avaliar os efeitos da radiação gama na qualidade físico-química, química e microbiológica da polpa de amora, armazenada sob refrigeração por 60 dias.

\section{MATERIAIS E MÉTODOS}

\section{Matéria-prima}

Os frutos de amora-preta foram adquiridos congelados da empresa De MARCHI, localizada em Jundiaí-SP.

\section{amora-preta}

\section{Processamento e obtenção da polpa de}

A obtenção da polpa de amora foi realizada na Planta-Piloto da Embrapa Agroindústria de Alimentos, no Rio de Janeiro. Os frutos foram selecionados, lavados e despolpados, com o auxílio de uma despolpadeira horizontal com uma peneira de $0,8 \mathrm{~mm}$ de diâmetro. Foram acondicionados em sacos plásticos de polietileno contendo $100 \mathrm{~g} /$ unidade e congelados $\left(-18^{\circ} \mathrm{C}\right)$ até o momento do processamento.

\section{Irradiação}

A irradiação da polpa de amora foi realizada no laboratório de irradiação gama do Centro de Desenvolvimento da Tecnologia Nuclear (CDTN), localizado em Belo Horizonte-MG. O laboratório conta com uma câmara irradiadora de $96 \mathrm{~m}^{2}$ com irradiador de cobalto 60 da MDS Nordion, Canadá, que mantém um padrão de segurança internacional para operação de irradiadores.

Com o objetivo de minimizar os efeitos da radiólise, a polpa de amora foi previamente congelada antes do processo de irradiação. Foram utilizados nove sacos por dose, totalizando 36 amostras de 100 $\mathrm{g}$ de polpa. A polpa foi submetida às seguintes doses: $0,75 \mathrm{kGy}, 1,5 \mathrm{kGy}, 3 \mathrm{kGy}$ e o controle. A taxa de dose emitida pela fonte de Cobalto- 60 foi de 3,24 kGy.h ${ }^{-1}$. Logo após o processo de irradiação gama, a polpa foi armazenada em uma temperatura de $4^{\circ} \mathrm{C}$, por 60 dias, sendo avaliadas nos tempos $0 ; 7 ; 15 ; 30$ e 60 dias. 


\section{Análises físico-químicas, químicas e micro- biológicas da polpa de amora}

A polpa de amora foi analisada quanto aos principais parâmetros químicos e físico-químicos. $\mathrm{O}$ $\mathrm{pH}$ e a acidez total foram determinados em $\mathrm{pHmetro}$ (Metrohm785 DMP Titrino), com leitura direta a $25^{\circ} \mathrm{C}$, e o teor de sólidos solúveis, em refratômetro do tipo Abbé (Bellingham + Stanley Limited), com escala em graus Brix. O teor de sólidos totais foi determinado a partir da determinação do peso seco, obtido pela secagem em estufa a vácuo a $60^{\circ} \mathrm{C}$ até a obtenção de peso constante (INSTITUTO ADOLFO LUTZ, 2008).

A concentração de antocianinas totais foi determinada pelo método do $\mathrm{pH}$ diferencial, de acordo Lee et al. (2008), que se baseia na obtenção de espectros das soluções em dois valores de $\mathrm{pH}$, visto que, com a alteração deste parâmetro, são observadas transformações nas estruturas das antocianinas e, consequentemente, na coloração das soluções (JACKMAN et al., 1987). Foram utilizadas soluções de $\mathrm{pH}$ 1,0 e 4,5 para a diluição do extrato, sendo as leituras realizadas em dois comprimentos de onda $(510 \mathrm{~nm}=$ absorção máxima; $700 \mathrm{~nm}=$ ausência de absorção). A absorbância das soluções a $510 \mathrm{~nm}$ em pH 1,0 é proporcional à concentração das antocianinas presentes, enquanto a absorbância das soluções neste mesmo comprimento de onda, porém em $\mathrm{pH} 4,5$, equivale aos produtos de degradação das antocianinas.

Para a determinação da atividade antioxidante, as amostras foram extraídas com metanol, conforme descrito por Rufino et al. (2010) e de quantificação de acordo com Re et al. (1999), baseada no método do ABTS expresso em TEAC (equivalente de Trolox).

Para a avaliação da cor, foi utilizado o colorímetro Color Meter-Minolta C-400, sendo realizadas três leituras sequenciais de cada amostra. A escala usada foi a de Hunter L*, $\mathrm{a}^{*}, \mathrm{~b}^{*}$, que mede as três dimensões da cor: $\mathrm{L}^{*}$ representa o eixo da luminosidade; $\mathrm{a}^{*}$ representa o eixo do vermelhoverde; $b^{*}$ representa o eixo do amarelo-azul.

As análises microbiológicas foram realizadas segundo os padrões preconizados pela legislação brasileira para polpas de frutas in natura e sucos de frutas, a qual se refere às determinações de coliformes e Salmonella. Para melhor avaliação das condições de higiene no processamento, foram realizadas as contagens de bolores e leveduras, a contagem total padrão em placas e a contagem total de bactéria psicotrópica (APHA, 2001).

Todas as análises foram realizadas em triplicata, e os resultados foram submetidos à análise estatística pelo Programa XLSTAT 7.5. Os resultados foram avaliados através da análise de variância ANOVA e pelo teste de Tukey, ambos ao nível de $1 \%$ de significância.

\section{RESULTADOS E DISCUSSÃO}

De acordo com os resultados da análise microbiológica da polpa de amora irradiada, podese verificar que não houve desenvolvimento de microrganismo nos tempos de 0 e 7 dias, em todos os tratamentos analisados.

O congelamento a que as amostras foram submetidas, para evitar o efeito da radiólise e a refrigeração durante o armazenamento, pode ter contribuído para esse resultado inicial, além da do processo da irradiação.

Tezotto-Uliana et al. (2013) avaliaram em seu trabalho a eficiência da radiação gama associada ao armazenamento refrigerado na framboesa in natura. Neste trabalho, utilizou-se a dose de 0,5; 1,0 e 2,0 kGy, e a temperatura de armazenamento foi de $0^{\circ} \mathrm{C}$. Foi possível verificar o efeito sinergético do armazenamento a frio com a irradiação que possibilitou o prolongamento da vida útil da framboesa em 8 dias.

A baixa temperatura contribuiu para a conservação da polpa de amora, e o congelamento utilizado para minimizar os efeitos da radiólise da água no momento da irradiação proporcionou um efeito sinérgico.

Byun et al. (2001) observaram que, após a irradiação, as células dos microrganismos são danificadas e gradativamente são inativadas. Esses danos aumentam a sensibilidade do microrganismo ao meio no qual o mesmo se encontra durante o armazenamento. Esse fenômeno é semelhante ao que ocorre com os alimentos tratados termicamente, ou seja, o microrganismo torna-se incapaz de reparar o dano causado e tende a morrer (LEISTNER, 1996).

Song et al.(2007) verificaram a influência da irradiação gama nas propriedades do suco de cenoura e de couve. A dose de $3 \mathrm{kGy}$ foi eficiente para eliminar bactérias aeróbias e coliformes do suco de cenoura; porém, para o suco de couve, foi necessária a dose de 5 kGy. No suco não irradiado, a contagem chegou a $10^{9}$ UFC. $\mathrm{ml}^{-1}$ após 3 dias de armazenamento a $10{ }^{\circ} \mathrm{C}$. O suco irradiado teve sua vida de prateleira prolongada por 3 dias, enquanto o não irradiado durou apenas 1 dia.

Youssef et al. (2011) avaliaram o efeito da irradiação gama em suco de tomate e constataram que a dose de irradiação de 3,0 kGy foi a que melhor garantiu a segurança microbiológica e prolongou a vida de prateleira do produto. O suco irradiado e 
armazenado a $4^{\circ} \mathrm{C}$ apresentou-se apto para consumo por mais 10 dias quando comparado ao suco não irradiado.

O tratamento com a dose de $1,5 \mathrm{kGy}$ estendeu a vida de prateleira até 60 dias e apresentou menor contagem para fungos filamentosos, leveduras e aeróbio psicrotróficos. Não foi detectada a presença de salmonella sp., e o número provável de coliformes

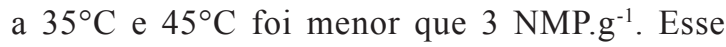
resultado indica que a amostra está apta para o consumo, em conformidade com o que preconiza a legislação brasileira.

A caracterização físico-química da polpa de amora irradiada ao longo dos 60 dias de armazenamento está apresentada na Tabela 1 .

Os valores de $\mathrm{pH}$ das amostras da polpa de amora-preta ficaram na faixa entre 2,89 e 2,99. Hirsch et al. (2012), ao fazerem a caracterização físico-química das variedades de amora-preta do Sul do Brasil, encontraram valores de $\mathrm{pH}$ na faixa entre 2,78 e 3,08. O resultado apresentado era o esperado devido às características naturais da fruta, que possui um sabor ácido a doce-ácido.

Não houve diferença significativa na acidez entre o controle e as amostras irradiadas nos tempos de armazenamento de 0; 7; 15 e 30 dias. Porém, aos 60 dias de armazenamento, ocorreu um aumento em todos os tratamentos. Os valores de acidez variaram de 1,53 a 1,73\%. Enquanto Hirsch et al. (2012) verificaram que a acidez de frutos de amora variou de $1,30 \%$ a 1,58\% de ácido cítrico. De acordo com Mota (2006), essa diferença na acidez dos frutos pode estar associada às condições edafoclimáticas do cultivo.

Não foi identificada alteração no teor de sólidos solúveis durante o armazenamento. Esse resultado também foi verificado por Zhao et al. (1996), em trabalho realizado com mamão, e por Domarco et al. (1999), no trabalho com uva Itália, no qual relatam que a radiação gama não exerceu efeito significativo no teor de sólidos solúveis das frutas irradiadas. Na amostra não irradiada, ocorreu redução no teor de sólido após 30 dias.

De acordo com os resultados referentes à análise de sólidos solúveis totais, sólidos solúveis e acidez, pode constatar-se que não houve interferência da radiação gama nos parâmetros físico-químicos da polpa de amora. Neves et al. (2002) mencionaram que a irradiação em frutos e hortaliças não ocasionou toxidez e praticamente manteve o valor nutritivo dos produtos quando comparados com aqueles processados por outros métodos.

Os valores do teor de antocianinas na polpa de amora irradiada estão apresentados na Tabela 2. Verificou-se que não ocorreu perda significativa no inicio do armazenamento, porém aos 60 dias de armazenamento houve uma redução no teor destes compostos. A antocianina é um pigmento instável, que pode ser degradado durante o processamento, congelamento e armazenamento.

Gonçalves et al. (2006) avaliaram a irradiação gama para a conservação da polpa de acerola e não constataram inicialmente perda no teor de antocianinas.

A avaliação da atividade antioxidante das amostras irradiadas durante os 60 dias de armazenamento está apresentada na Tabela 3. Os valores de atividade antioxidante mantiveram-se superiores a $27,8 \mu \mathrm{mol} . \mathrm{g}^{-1}$ durante todo o período avaliado.

Silva et al. (2011) avaliaram a capacidade antioxidante pelo método DPPH e ABTS em frutas produzidas na região do Rio Grande do Sul. Para as cultivares de amora-preta, encontraram os seguintes valores: a cultivar Xavante (13,22 e 16,52 $\mu$ mols. $\left.\mathrm{g}^{-1}\right)$,

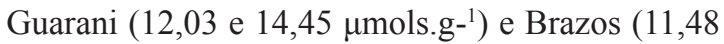
e 13,44 $\mu$ mols. $\left.\mathrm{g}^{-1}\right)$. De acordo com esses autores, existe uma correlação entre o teor de antocianinas e o valor da capacidade antioxidante dos frutos de amora-preta.

Em estudo sobre os aspectos quantitativos da degradação de antocianinas em morango, Markakis, Livinngston e Fellers (1957) observaram que a reação hidrolítica responsável pela degradação do pigmento apresentou relação direta com a temperatura de estocagem, o que indicaria a necessidade de utilizar a menor temperatura possível em todas as etapas de processamento e armazenamento.

A avaliação de cor realizada na polpa de amora irradiada está apresentada na Tabela 4 . O valor de L* variou entre 15,70 e 19.44 , indicando que a polpa de amora possui coloração escura.

A coordenada $\mathrm{a}^{*}$ indica a intensidade do vermelho ao verde (valores positivos são tons de vermelho, e valores negativos, tons de verde). $\mathrm{O}$ valor de $\mathrm{a}^{*}$ permaneceu sempre positivo, indicando que a polpa de amora apresentou cor característica vermelha.

De acordo com Lopez et al. (2000), as antocianinas são diretamente influenciadas pela substituição dos grupos hidroxila e metoxila na molécula. Com o incremento do número de grupos hidroxilas, as mesmas tendem a apresentar coloração azulada. Com relação à estabilidade das antocianinas, as mesmas se encontram comumente na forma de uma mistura de diferentes estruturas químicas em equilíbrio: cátion flavilium (vermelho), base anidra quinoidal (azul), pseudobase carbitol (incolor) e chalcona (incolor ou levemente amarela). 
TABELA 1- Avaliação físico-química da polpa de amora processada por irradiação em função da dose aplicada ao longo do armazenamento.

\begin{tabular}{|c|c|c|c|c|}
\hline \multicolumn{5}{|c|}{ Dose (kGy) } \\
\hline \multicolumn{5}{|c|}{ pH } \\
\hline $\begin{array}{c}\text { Tempo } \\
\text { (dias) }\end{array}$ & $\mathbf{0}$ & 0,75 & 1,5 & 3,0 \\
\hline 0 & $2,91 \pm 0,02$ & $2,94 \pm 0,01$ & $2,95 \pm 0,01$ & $2,93 \pm 0,02$ \\
\hline 7 & $2,92 \pm 0,02$ & $2,92 \pm 0,04$ & $2,92 \pm 0,01$ & $2,94 \pm 0,02$ \\
\hline 15 & $2,91 \pm 0,02$ & $2,92 \pm 0,02$ & $2,92 \pm 0,02$ & $2,94 \pm 0,01$ \\
\hline 30 & $2,96 \pm 0,00$ & $2,99 \pm 0,01$ & $2,96 \pm 0,01$ & $2,96 \pm 0,01$ \\
\hline 60 & $2,89 \pm 0,02$ & $2,89 \pm 0,00$ & $2,89 \pm 0,01$ & $2,92 \pm 0,01$ \\
\hline \multicolumn{5}{|c|}{$\begin{array}{l}\text { Acidez Total Titulável } \\
\text { (\% ácido cítrico) }\end{array}$} \\
\hline $\mathbf{0}$ & $1,57 \mathrm{Aa} \pm 0,00$ & $1,67 \mathrm{Aa} \pm 0,01$ & $1,59 \mathrm{Aa} \pm 0,01$ & $1,53 \mathrm{Aa} \pm 0,03$ \\
\hline 7 & $1,70 \mathrm{Aa} \pm 0,07$ & $1,66 \mathrm{Aa} \pm 0,01$ & $1,67 \mathrm{Aa} \pm 0,02$ & $1,63 \mathrm{Aa} \pm 0,03$ \\
\hline 15 & $1,58 \mathrm{Aa} \pm 0,01$ & $1,67 \mathrm{Aa} \pm 0,07$ & $1,64 \mathrm{Aa} \pm 0,05$ & $1,63 \mathrm{Aa} \pm 0,00$ \\
\hline 30 & $1,60 \mathrm{Aa} \pm 0,03$ & $1,63 \mathrm{Aa} \pm 0,00$ & $1,62 \mathrm{Aa} \pm 0,02$ & $1,63 \mathrm{Aa} \pm 0,02$ \\
\hline 60 & $1,62 \mathrm{Aa} \pm 0,01$ & $1,71 \mathrm{Bab} \pm 0,11$ & $1,73 \mathrm{Bb} \pm \mathbf{0}, \mathbf{1 7}$ & $1,66 \mathrm{ABab} \pm \mathbf{0 , 0 8}$ \\
\hline \multicolumn{5}{|c|}{$\begin{array}{l}\text { Sólidos Solúveis Totais } \\
\left({ }^{\circ} \text { Brix }\right)\end{array}$} \\
\hline $\mathbf{0}$ & $8,0 \mathrm{Bc}$ & $8,0 \mathrm{Bc}$ & $8,0 \mathrm{Bc}$ & $8,0 \mathrm{Bc}$ \\
\hline 7 & $7,5 \mathrm{Ab}$ & $8,0 \mathrm{Bc}$ & $8,0 \mathrm{Bc}$ & $8,0 \mathrm{Bc}$ \\
\hline 15 & $8,0 \mathrm{Bc}$ & $8,0 \mathrm{Bc}$ & $8,0 \mathrm{Bc}$ & $8,0 \mathrm{Bc}$ \\
\hline 30 & 7,5 Ab & $8,0 \mathrm{Bc}$ & $8,0 \mathrm{Bc}$ & $8,0 \mathrm{Bc}$ \\
\hline 60 & 7,0 Aa & $8,0 \mathrm{Bc}$ & $8,0 \mathrm{Bc}$ & $8,0 \mathrm{Bc}$ \\
\hline \multicolumn{5}{|c|}{$\begin{array}{l}\text { Sólidos Totais } \\
(\mathrm{g} / \mathbf{1 0 0 g})\end{array}$} \\
\hline $\mathbf{0}$ & $8,35 \mathrm{BCa} \pm 0,20$ & $8,30 \mathrm{Aa} \pm 0,05$ & $7,96 \mathrm{Aa} \pm 0,06$ & $8,48 \mathrm{Ba} \pm 0,30$ \\
\hline 7 & $7,86 \mathrm{Aa} \pm 0,12$ & $8,28 \mathrm{Aa} \pm 0,09$ & $8,35 \mathrm{Aa} \pm 0,17$ & $8,07 \mathrm{ABa} \pm 0,16$ \\
\hline 15 & $8,11 \mathrm{ABab} \pm 0,09$ & $8,40 \mathrm{Ab} \pm 0,27$ & $8,07 \mathrm{Aab} \pm 0,08$ & $7,52 \mathrm{Aa} \pm 0,07$ \\
\hline 30 & $8,58 \mathrm{Ca} \pm 0,10$ & $8,57 \mathrm{Aa} \pm 0,11$ & $8,53 \mathrm{Aa} \pm 0,14$ & $8,68 \mathrm{Ba} \pm 0,03$ \\
\hline 60 & $8,52 \mathrm{BCa} \pm 0,10$ & $8,26 \mathrm{Aa} \pm 0,20$ & $8,21 \mathrm{Aa} \pm 0,75$ & $7,48 \mathrm{Aa} \pm 0,11$ \\
\hline
\end{tabular}

Efeito do tempo de armazenamento - Médias \pm Desvio-padrão seguidas por letras maiúsculas iguais na mesma linha não diferem entre si, pelo teste de Tukey, a $1 \%$ de probabilidade. Efeito da dose - Médias \pm Desvio-padrão seguidas por letras minúsculas iguais na mesma coluna não diferem entre si, pelo teste de Tukey, a $1 \%$ de probabilidade 
TABELA 2 - Análises do teor de antocianinas (mg/100g) na polpa de amora irradiada .

\begin{tabular}{lccccc}
\hline Dose/ Tempo & $\mathbf{0 ~ d i a}$ & $\mathbf{7 ~ d i a s}$ & $\mathbf{1 5}$ dias & 30 dias & 60 dias \\
\hline $\mathbf{0}$ & $28,82 \mathrm{Ab} \pm 0,43$ & $28,67 \mathrm{Ab} \pm 0,74$ & $27,23 \mathrm{Ab} \pm 0,41$ & $23,44 \mathrm{Aa} \pm 0,36$ & $23,61 \mathrm{Ba} \pm 0,22$ \\
$\mathbf{0 , 7 5} \mathbf{k G y}$ & $27,50 \mathrm{Ab} \pm 0,39$ & $27,98 \mathrm{Ab} \pm 0,20$ & $27,32 \mathrm{Ab} \pm 0,10$ & $27,78 \mathrm{Bb} \pm 0,91$ & $23,61 \mathrm{Ba} \pm 0,47$ \\
$\mathbf{1 , 5} \mathbf{k G y}$ & $27,68 \mathrm{Ab} \pm 0,83$ & $28,92 \mathrm{Ab} \pm 0,47$ & $28,27 \mathrm{Ab} \pm 0,81$ & $28,76 \mathrm{Bb} \pm 0,16$ & $24,18 \mathrm{Ba} \pm 0,42$ \\
$\mathbf{3 , 0} \mathbf{k G y}$ & $28,93 \mathrm{Ac} \pm 0,37$ & $27,43 \mathrm{Abc} \pm 0,97$ & $26,71 \mathrm{Abc} \pm 0,78$ & $25,11 \mathrm{Ab} \pm 1,05$ & $20,45 \mathrm{Aa} \pm 0,02$ \\
\hline
\end{tabular}

Efeito da dose - Médias \pm desvio-padrão seguidas por letras maiúsculas iguais na mesma coluna não diferem entre si, pelo teste de Tukey, a $1 \%$ de probabilidade. Efeito do tempo de armazenamento - Médias seguidas por letras minúsculas iguais na mesma linha não diferem entre si, pelo teste de Tukey, a $1 \%$ de probabilidade.

TABELA 3 - Análises de atividade antioxidante $(\mu \mathrm{mol} / \mathrm{g})$ da polpa de amora irradiada,

\begin{tabular}{cccccc}
\hline Dose/ Tempo & $\mathbf{0 ~ d i a}$ & $\mathbf{7 ~ d i a s}$ & $\mathbf{1 5}$ dias & 30 dias & 60 dias \\
\hline $\mathbf{0}$ & $33,13 \mathrm{Ab} \pm 0,77$ & $27,82 \mathrm{Aa} \pm 0,71$ & $29,21 \mathrm{Aab} \pm 1,59$ & $32,12 \mathrm{Aab} \pm 1,50$ & $31,95 \mathrm{Aab} \pm 1,35$ \\
$\mathbf{0 , 7 5} \mathbf{~ k G y}$ & $32,39 \mathrm{Aa} \pm 0,89$ & $29,92 \mathrm{Aa} \pm 0,93$ & $29,28 \mathrm{Aa} \pm 1,46$ & $31,83 \mathrm{Aa} \pm 0,70$ & $29,58 \mathrm{Aa} \pm 0,58$ \\
$\mathbf{1 , 5} \mathbf{k G y}$ & $30,93 \mathrm{Aa} \pm 0,47$ & $30,74 \mathrm{Aa} \pm 0,31$ & $29,40 \mathrm{Aa} \pm 1,31$ & $31,98 \mathrm{Aa} \pm 1,00$ & $32,62 \mathrm{Aa} \pm 0,24$ \\
$\mathbf{3 , 0} \mathbf{k G y}$ & $28,60 \mathrm{Aab} \pm 1,77$ & $29,61 \mathrm{Aab} \pm 0,60$ & $28,28 \mathrm{Aa} \pm 0,59$ & $30,15 \mathrm{Aab} \pm 0,49$ & $32,41 \mathrm{Ab} \pm 1,15$ \\
\hline
\end{tabular}

Efeito da dose - Médias \pm desvio-padrão seguidas por letras maiúsculas iguais na mesma coluna não diferem entre si, pelo teste de Tukey, a $1 \%$ de probabilidade. Efeito do tempo de armazenamento - Médias \pm desvio-padrão seguidas por letras minúsculas iguais na mesma linha não diferem entre si, pelo teste de Tukey, a $1 \%$ de probabilidade.

TABELA 4 - Avaliação de cor da polpa de amora irradiada.

\begin{tabular}{|c|c|c|c|c|c|}
\hline & & & $* \mathbf{L}$ & & \\
\hline Dose/ Tempo & 0 dia & 7 dias & 15 dias & 30 dias & 60 dias \\
\hline $\mathbf{0}$ & $18,73 \mathrm{~A}$ & $18,32 \mathrm{~B}$ & $19,27 B$ & $17,15 \mathrm{~A}$ & $15,70 \mathrm{~A}$ \\
\hline 0,75 kGy & $20,51 \mathrm{~A}$ & $18,96 \mathrm{~B}$ & $19,84 \mathrm{~B}$ & $18,97 \mathrm{~A}$ & $17,80 \mathrm{C}$ \\
\hline 1,5 kGy & $17,71 \mathrm{~A}$ & $18,68 B$ & $17,29 \mathrm{~A}$ & $18,17 \mathrm{~A}$ & $17,92 \mathrm{C}$ \\
\hline \multirow[t]{2}{*}{ 3,0 kGy } & $19,44 \mathrm{~A}$ & $15,75 \mathrm{~A}$ & $18,88 \mathrm{~A}$ & $18,75 \mathrm{~A}$ & $16,77 \mathrm{~B}$ \\
\hline & \multicolumn{5}{|c|}{$a^{*}$} \\
\hline Dose/ Tempo & 0 dia & 7 dias & 15 dias & 30 dias & 60 dias \\
\hline $\mathbf{0}$ & $21,56 \mathrm{AB}$ & $21,78 \mathrm{~B}$ & $21,64 \mathrm{AB}$ & $22,43 \mathrm{~A}$ & $19,01 \mathrm{~A}$ \\
\hline 0,75 kGy & $22,93 \mathrm{~B}$ & $22,43 B$ & $23,55 \mathrm{~B}$ & $20,27 \mathrm{~A}$ & $21,46 \mathrm{~B}$ \\
\hline 1,5 kGy & $19,25 \mathrm{~A}$ & $22,68 B$ & $21,44 \mathrm{AB}$ & $22,00 \mathrm{~A}$ & $20,62 \mathrm{~B}$ \\
\hline \multirow[t]{2}{*}{ 3,0 kGy } & $21,67 \mathrm{AB}$ & $19,38 \mathrm{~A}$ & $17,70 \mathrm{~A}$ & $22,06 \mathrm{~A}$ & $19,30 \mathrm{~A}$ \\
\hline & \multicolumn{5}{|c|}{$\mathbf{b}^{*}$} \\
\hline Dose/ Tempo & 0 dia & 7 dias & 15 dias & 30 dias & 60 dias \\
\hline $\mathbf{0}$ & $-2,56 \mathrm{~A}$ & $-1,74 \mathrm{~B}$ & $-0,80 \mathrm{~B}$ & $-2,24 \mathrm{~A}$ & $-3,80 \mathrm{~A}$ \\
\hline 0,75 kGy & $-2,28 \mathrm{~A}$ & $-1,30 \mathrm{~B}$ & $-0,75 \mathrm{~B}$ & $-1,65 \mathrm{~A}$ & $-2,20 \mathrm{~B}$ \\
\hline 1,5 kGy & $-4,55 \mathrm{~A}$ & $-1,28 \mathrm{~B}$ & $-4,66 \mathrm{~A}$ & $-2,11 \mathrm{~A}$ & $-2,38 \mathrm{C}$ \\
\hline \multirow[t]{2}{*}{$3,0 \mathrm{kGy}$} & $-2,39 \mathrm{~A}$ & $-3,44 \mathrm{~A}$ & $-2,50 \mathrm{AB}$ & $-2,02 \mathrm{~A}$ & $-3,20 \mathrm{C}$ \\
\hline & \multicolumn{5}{|c|}{$\Delta \mathbf{E}$} \\
\hline Dose/ Tempo & 0 dia & 7 dias & 15 dias & 30 dias & 60 dias \\
\hline $\mathbf{0}$ & $28,68 \mathrm{AB}$ & $28,51 \mathrm{~B}$ & 29,04B & $28,34 \mathrm{~A}$ & $24,95 \mathrm{~A}$ \\
\hline 0,75 kGy & $30,86 \mathrm{~B}$ & $29,40 B$ & $30,81 \mathrm{~B}$ & $27,86 \mathrm{~A}$ & $27,97 \mathrm{~B}$ \\
\hline 1,5 kGy & $26,57 \mathrm{~A}$ & $29,41 B$ & $25,19 \mathrm{~A}$ & $28,61 \mathrm{~A}$ & $27,43 \mathrm{~B}$ \\
\hline 3,0 kGy & $29,23 \mathrm{AB}$ & $25,21 \mathrm{~A}$ & $28,68 B$ & $29,03 \mathrm{~A}$ & $25,77 \mathrm{~A}$ \\
\hline
\end{tabular}

Médias seguidas por letras iguais na mesma coluna não diferem entre si, pelo teste de Tukey, a 1\% de probabilidade. L - luminosidade $(0=$ preto e $100=$ branco $)$ a $-(-80$ até zero $=$ verde; do zero ao $+100=$ vermelho $) ; b-(-100$ até zero $=$ azul; do zero ao $+70=$ amarelo $)$. 


\section{CONCLUSÃO}

Pode-se concluir que o tratamento de 1,5 kGy foi o que apresentou a menor contagem de microrganismo e garantiu a qualidade microbiológica por mais tempo, ou seja, 60 dias de armazenamento sob refrigeração. Além disso, manteve-se apta ao consumo e em conformidade com o que determina a legislação brasileira vigente. Foi observado que, durante todo o período de armazenamento, a acidez, $\mathrm{pH}$, teor de sólidos solúveis e teor de sólidos totais permaneceram sem grandes modificações, mostrando dessa maneira que não houve interferência da irradiação gama nos parâmetros físico-químicos durante o tempo analisado. Porém, o armazenamento foi responsável pelas diferenças observadas nos parâmetros avaliados. Em todas as faixas de irradiação avaliada, a concentração de antocianinas e a atividade antioxidante foram mantidas até os 30 dias de armazenamento.

\section{AGRADECIMENTOS}

Os autores agradecem ao Centro de Desenvolvimento da Tecnologia Nuclear (CDTN), pela disponibilização do irradiador; à Prof ${ }^{a}$. Dra. Louise Emy Kurozawa, da Universidade Federal Rural do Rio de Janeiro, pelas sugestões durante a elaboração deste artigo, e à Coordenação de Aperfeiçoamento de Pessoal de Nível Superior (CAPES), pela bolsa de mestrado.

\section{REFERÊNCIAS}

ALAM KHAN, K.; ABRAHEM, M. Effect of irradiation on quality of spices. International Food Research Journal, Selangor, v.17, p. 825-836, 2010.

ANTUNES, L. E. C.; GONÇALVES, E. D.; TREVISAN, R. Alterações da atividade da poligalacturonase e pectinametilesterase em amorapreta (rubus spp.) durante o armazenamento. Revista Brasileira Agrociência, Pelotas, v.12, n.1, p.63-66, 2006.

APHA. American Public Health Association. Committee on Microbiological for foods. Compendium of methods for the microbiological examination of foods. 4.ed. Washington: American Public Health Association, 2001. 676p.
BOWEN-FORBES, C. S.; ZHANG, Y.; NAIR, M. G. Anthocyanin content, antioxidant, anti-inflammatory and anticancer properties of blackberry and raspberry fruits. Journal of Food Composition and Analysis, San Diego, v.23, n.6, p.554-560, 2010.

BYUN, M.; KIM, D.; YOOK, H. S.; CHA, B. S.; KIM, J. O. Changes in microbiological and general qualities in gamma irradiated Doenjang (fermented soybean paste). Food Science and Biotechnology, Boca Raton, v.10, p.7-11, 2001.

DOMARCO, R. E.; SPOTO, M. H. F.; BLUMER, L.; WALDER, J. M. M. Sinergia da radiação ionizante e do aquecimento na vida de prateleira da uva "Itália". Scientia Agricola, Piracicaba, v.56, n.4, p.981-986, 1999.

FARKAS, J. Irradiation for better foods. Trends in Food Science \& Technology, Cambridge, v.17, n.4, p.148-152, 2006.

FARKAS, J.; MOHÁCSI-FARKAS, C. History and future of food irradiation. Trends in Food Science \& Technology, Cambridge, v.22, n.2-3, p.121-126, 2011.

GONÇALVES, M. P. J. C.; BOTREL, D. A.; SOARES, N. F. F.; STRINGHETA, P. C. Irradiação gama como alternativa de conservação de polpa de acerola. Alimentação e Nutrição, Araraquara. v. 17, n. 2, p.159-163, 2006.

HIRSCH, G. E.; FACCO, E. M. P.; RODRIGUES, D. B.; VIZZOTTO, M.; EMANUELli, T. Caracterização físico-química de variedades de amora-preta da região Sul do Brasil. Ciência Rural, Santa Maria, v.42, n.5, 2012.

INSTITUTO ADOLFO LUTZ. Métodos físicoquímicos para análise de alimentos. 4. ed. São Paulo: Instituto Adolfo Lutz, 2008. p.1020.

JACKMAN, R. L.; YADA, R. Y.; TUNG, M. A.; SPEERS, R. A. Anthocyanins as food colorants. A Review. Journal of Food Biochemistry, Westport, v.11, p.201-247, 1987.

JACQUES, A. C.; PERTUZATTI, P. B.; BARCIA, M. T.; ZAMBIAZI, R. C.; CHIM, J. F. Estabilidade de Compostos Bioativos em Polpa Congelada de Amora-Preta (rubus fruticosus) cv.tupy. Química Nova, São Paulo, v.33, n.8, p.1720-1725, 2010. 
LEE, J.; RENNAKER, C.; WROLSTAD, R. E. Correlation of two anthocyanin quantification method: HPLC and spectrophotometric methods. Food Chemistry, London, v.110, p.782-786, 2008.

LEISTNER, L. Food protection by hurdle technology. Journal of Food Protection, Des Moines, v.2, p.2-27, 1996.

LÓPEZ, O.P.; JIMÉNEZ, A.R.; VARGAS, F.D. et al. Natural pigments: carotenoids, anthocyanins, and betalains - characteristics, biosynthesis, processing, and stability. Critical Reviews in Food Science and Nutrition, Boca Raton, v. 40, n. 3, p.173-289, 2000.

MARKAKIS, P.; LIVINGSTON, G. E.; FELLERS, C. R. Quantitative aspects of strawberry pigment degradation. Food Research, Chicago, v. 22, p. 117-130, 1957.

MOTA, R. V. Caracterização do suco de amora-preta elaborado em extrator caseiro. Ciência e Tecnologia de Alimentos, Campinas, v.26, n.2, p.303-308, 2006.

NEVES, L. C.; MANZIONE, R. L.; VIEITES, R. L. Radiação gama na conservação pós-colheita da nectarina (Prunus persica var. Nucipersica) frigoconservada. Revista Brasileira de Fruticultura, Jaboticabal, v.24, n.3, 2002.

RE, R.; PELLEGRINI, N.; PROTEGGENTE, A.; PANNALA, A.; YANG, M.; RICE-EVANS, C. Antioxidant activity applying an improved abts radical cation decolorization assay. Free Radical Biology \& Medicine, New York, v.26, n.9-10, p.1231-1237, 1999.

RUFINO, M. S. M.; ALVES, R. E.; BRITO, E. S.; PÉREZ-JIMÉNEZ, J.; SAURA-CALIXTO, F.; MANCINI-FILHO, J. Bioactive compounds and antioxidant capacities of 18 non traditional tropical fruits from Brazil. Food Chemistry, Chicago, v.121, p.996-1002, 2010.
SCHAKER, P. D. C.; ANTONIOLLI, L. R. Aspectos econômicos e tecnológicos em pós-colheita de amoras-pretas (Rubus spp). Revista Brasileira de Agrociência, Pelotas, v.15, n.1/4, p.11-15, 2009.

SILVA, R. S.; VENDRUSCOLO, J. L.; TORALLES, R. P. Avaliação da capacidade antioxidante em frutas produzidas na região sul do RS. Revista Brasileira de Agrociência, Pelotas, v.17, n.3-4, p.392-400, 2011.

SONG, H.; BYUN, M.; JO, C.; LEE, C.; KIM, K.; KIM, D. Effects of gamma irradiation on the microbiological, nutritional, and sensory properties of fresh vegetable juice. Food Control, Guildford, v.18, p.5-10, 2007.

TEZOTTO-ULIANA, J. V.; BERNO, N. D.; SAJI, F. R. Q.; KLUGE, R. A. Gamma radiation: An efficient technology to conserve the quality of fresh raspberries. Scientia Horticulturae, Amsterdam, n. 164, p.348-352, 2013.

WU, R.; FREI, B.; KENNEDY, J. A.; ZHAO, Y. Effects of refrigerated storage and processing technologies on the bioactive compounds and antioxidant capacities of 'Marion' and 'Evergreen' blackberries. Food Science and Technology, London, v. 43, p. 1.253-1.264, 2010.

YOUSSEF, K. A.; HAMMAD, A. I.; ABD ELKALEK, H. H.; ABD EL-KADER, R. M. Ensure Microbial Safety and Extending Shelf-Life of Tomato Juice by $\gamma$ Irradiation. Nature and Science, New York, v. 11, p. 154-163, 2011.

ZHAO, M.; MOY, J.; PAULL, R. E. Effect of gamma-irradiation on ripening papaya pectin. Postharvest Biology and Technology, Amsterdam, v.8, p.209-222, 1996. 